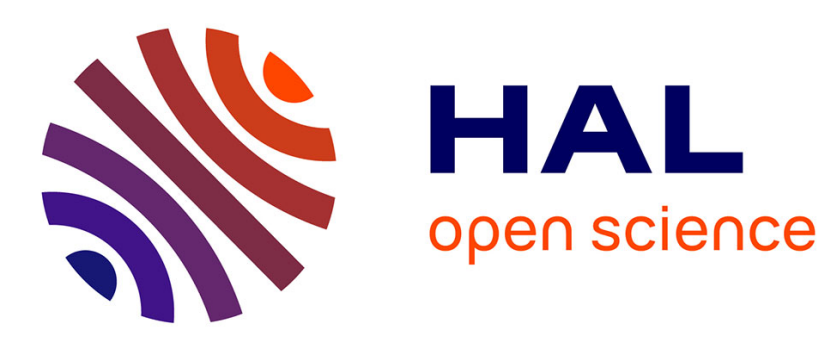

\title{
On the deformation of gas bubbles in liquids
} Dominique Legendre, Roberto Zenit, Rodrigo Velez-Cordero

\section{To cite this version:}

Dominique Legendre, Roberto Zenit, Rodrigo Velez-Cordero. On the deformation of gas bubbles in liquids. Physics of Fluids, 2012, vol. 24, 10.1063/1.4705527 . hal-00921321

\section{HAL Id: hal-00921321 https://hal.science/hal-00921321}

Submitted on 20 Dec 2013

HAL is a multi-disciplinary open access archive for the deposit and dissemination of scientific research documents, whether they are published or not. The documents may come from teaching and research institutions in France or abroad, or from public or private research centers.
L'archive ouverte pluridisciplinaire $\mathbf{H A L}$, est destinée au dépôt et à la diffusion de documents scientifiques de niveau recherche, publiés ou non, émanant des établissements d'enseignement et de recherche français ou étrangers, des laboratoires publics ou privés. 


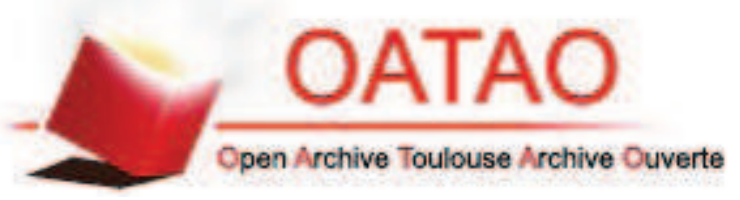

OATAO is an open access repository that collects the work of Toulouse researchers and makes it freely available over the web where possible.

This is an author-deposited version published in : http://oatao.univ-toulouse.fr/ Eprints ID : 10534

To link to this article : DOI: $10.1063 / 1.4705527$

http://dx.doi.org/10. 1063/1.4705527

To cite this version Legendre, Dominique and Zenit, Roberto and VelezCordero, Rodrigo On the deformation of gas bubbles in liquids. (2012) Physics of Fluids, vol. 24 . ISSN 1070-6631

Any correspondance concerning this service should be sent to the repository administrator: staff-oatao@ listes-diff.inp-toulouse.fr 


\title{
On the deformation of gas bubbles in liquids
}

\author{
Dominique Legendre, ${ }^{1}$ Roberto Zenit, ${ }^{2}$ and J. Rodrigo Velez-Cordero ${ }^{2}$ \\ ${ }^{1}$ Université de Toulouse, INPT, UPS, CNRS, IMFT (Institut de Mécanique des Fluides de \\ Toulouse), Allée Camille Soula, F-31400 Toulouse, France \\ ${ }^{2}$ Instituto de Investigaciones en Materiales, Universidad Nacional Autónoma de México, \\ Apdo. Postal 70-360, Cd. Universitaria, México D.F. 04510, México
}

\begin{abstract}
We consider the deformation of gas bubbles rising in different liquids over a wide range of Morton numbers, from $\mathrm{O}\left(10^{-11}\right)$ to $\mathrm{O}(1)$, and bubble diameters. We have collected data from the literature and performed new experiments for relatively large Morton numbers. A simple expression is proposed to describe the evolution of the bubble deformation, which is consistent with the analytical solution of Moore ["The rise of a gas bubble in a viscous liquid," J. Fluid Mech. 6, 113 (1959)]. It appears that deformation can be predicted correctly by considering the Morton and Weber numbers. The variation of the bubble interfacial area is also analyzed; this quantity is very important for the case of bubbly flow modeling but has not been measured directly to date.
\end{abstract}

\section{INTRODUCTION}

In air water systems millimetric bubbles are known to deform. ${ }^{1}$ The deformation changes the bubble hydrodynamics and characteristic trajectories of rising bubbles; these issues have been recently studied in detail by Ellingsen and Risso, ${ }^{2}$ Sanada et al. ${ }^{3}$ Mougin and Magnaudet, ${ }^{4}$ Zenit and Magnaudet, ${ }^{5}$ and Yang and Prosperetti. ${ }^{6}$ The effect of deformation on the drag coefficient has also been considered (Moore, ${ }^{7}$ Maxworthy et al. ${ }^{8}$ Tomiyama et al.,${ }^{9}$ and Legendre ${ }^{10}$ ). As a consequence of hydrodynamic modifications, bubble deformation also impacts the interfacial heat or mass transfer (Lochiel and Calderbank, ${ }^{11}$ Favelukis and Ly, ${ }^{12}$ and Figueroa and Legendre ${ }^{13}$ ). The modeling of bubble deformation is of great importance in bubbly flows since the deformation results in a bigger interfacial area for the mass, momentum, and energy transfer between the phases. For practical applications, it is thus necessary to use a relevant model to characterize bubble deformation.

In this investigation, we have collected data from the numerous sources in the specialized literature; we have also conducted experiments for a particular range of Morton numbers for which no detailed, or reliable, information could be found. We propose a simple expression to predict the bubble deformation. We also propose this expression to be used for the calculation of the interfacial surface area of air bubbles in liquids.

This note is organized as follows. Section II presents the problem considered. The experimental device is described in Sec. III. Section IV considers bubble deformation in air water system and the effect of bubble contamination is discussed. Section V presents the effect of increasing the fluid viscosity; a simple model able to describe bubble deformation for a wide range of Morton numbers is proposed in Sec. VI. Finally, results are used to discuss the evolution of the interfacial area in Sec. VII.

\section{STATEMENT OF THE PROBLEM}

Let us consider a gas bubble of constant volume $V$ rising at its terminal velocity $\mathbf{U}$ in a liquid at rest under the action of gravity $g$. The viscosity and density of the liquid are denoted by $\rho$ and $\mu$, respectively. $\sigma$ is the interfacial surface tension and $d=(6 \mathrm{~V} / \pi)^{1 / 3}$ is the spherically equivalent diameter. The bubble deformation is first characterized by considering the aspect ratio of a bubble, 
TABLE I. Physical properties of the water-glycerin (W-G) solutions used in this study.

\begin{tabular}{lcccc}
\hline \hline $\begin{array}{l}\text { Fluid } \\
\text { water-glycerin ratio (v/v) }\end{array}$ & $\begin{array}{c}\text { Density } \\
\left(\mathrm{kg} / \mathrm{m}^{3}\right)\end{array}$ & $\begin{array}{c}\text { Viscosity } \\
(\mathrm{mPa} \mathrm{s})\end{array}$ & $\begin{array}{c}\text { Surface tension } \\
(\mathrm{mN} / \mathrm{m})\end{array}$ & Morton number \\
\hline Water (reference) & 1000 & 1.0 & 72.5 & $2.5 \times 10^{-11}$ \\
$25 / 75 \mathrm{~W}-\mathrm{G}$ & 1191 & 19.9 & 65.7 & $4.6 \times 10^{-6}$ \\
$11 / 89 \mathrm{~W}-\mathrm{G}$ & 1225 & 80.0 & 62.7 & $1.3 \times 10^{-3}$ \\
$8 / 92 \mathrm{~W}-\mathrm{G}$ & 1242 & 173.5 & 62.6 & $2.9 \times 10^{-2}$ \\
$4 / 96 \mathrm{~W}-\mathrm{G}$ & 1258 & 457.8 & 58.8 & $1.7 \times 10^{0}$ \\
\hline \hline
\end{tabular}

$\chi=a / b$, where $a$ and $b$ are the lengths in the perpendicular and parallel directions (with respect to the bubble velocity direction), respectively. Since bubbles are flattened due to their motion, we have $\chi \geq 1$. In Sec. VII, the deformation is characterized by considering the normalized bubble surface $f_{\alpha}=S / \pi d^{2}$ using the surface of the sphere of same volume. $f_{\alpha}$ thus expresses the relative increase of interface due to the deformation.

In this note we consider gas bubbles in liquids; hence, the bubble density and viscosity have a negligible effect on bubble dynamics and deformation. Considering the variables involved in the problem (namely, $d, \rho, \sigma, \mu$, and $g$ ), by simple dimensional analysis, we can show that the bubble aspect ratio $\chi$ and the normalized bubble interface $f_{\alpha}$ depend only on two independent dimensionless numbers. Several can be formed, but the most commonly found in this subject are: the bubble Reynolds number $R e=\rho U d / \mu$, the Weber number $W e=\rho U^{2} d / \sigma$, the Eötvös number $E o=\rho g d^{2} / \sigma$, and the Morton number $M o=\mu^{4} g / \sigma^{3} \rho$, to name a few.

\section{EXPERIMENTAL ARRANGEMENT}

Although there is a vast experimental database in the literature, we identified a wide range of Morton numbers for which no detailed information, for the particular case of significant bubble deformation, could be found. Hence, we conducted some simple experiments considering four different water-glycerin mixtures corresponding to $M o=1.24, M o=3.0 \times 10^{-2}, M o=1.4 \times 10^{-4}$, and $M o=1.6 \times 10^{-6}$. The physical properties of these liquids are shown in Table I. The viscosity of the liquids was measured with a rheometer (TA Instruments AR1000N) with a cone-plate geometry (60 mm, $2^{\circ}$, a gap of $65 \mu \mathrm{m}$ ). The surface tension measurements were performed with a DuNouy ring (diameter of $19.4 \mathrm{~mm}$, KSV Sigma 70). The density was measured with a pycnometer (Simax, $50 \mathrm{ml})$.

The bubbles were released in a cylindrical glass tube with a diameter $D$ of $9 \mathrm{~cm}$; a detailed description of the device to release the bubbles can be found in Soto et al. ${ }^{14}$ The ratio $d / D$ was less than 0.08 for all the experiments. The motion of the bubbles was captured using a high speed camera (MotionScope PCI 8000s, 500 frames/s). The resolution of the images was in the order of 15 pixels $/ \mathrm{mm}$, and varied only slightly for the different experiments. The camera was placed around $90 d$ above the base of the tube where the bubbles were released to ensure that the bubbles had reached their terminal properties; the measurement region was about $30 \mathrm{~d}$ below the liquid surface.

Some typical bubble shapes are shown in Fig. 1. We can observe that the deformation is significantly affected by the value of the Morton number. The shape evolves from spherical, to ellipsoidal and to spherical cap as both the Morton number and the bubble size increase.

\section{A. Digital image analysis}

The shape and position of the bubbles were determined by digital image analysis (DIA), using the Image Processing Toolbox of MATLAB. The terminal velocity was calculated from the displacement of the geometric center of bubble in time. The bubble aspect ratio, $\chi$, was determined from the direct measurement of $a$ and $b$. Figure 2 shows a typical image of a bubble and its corresponding image 


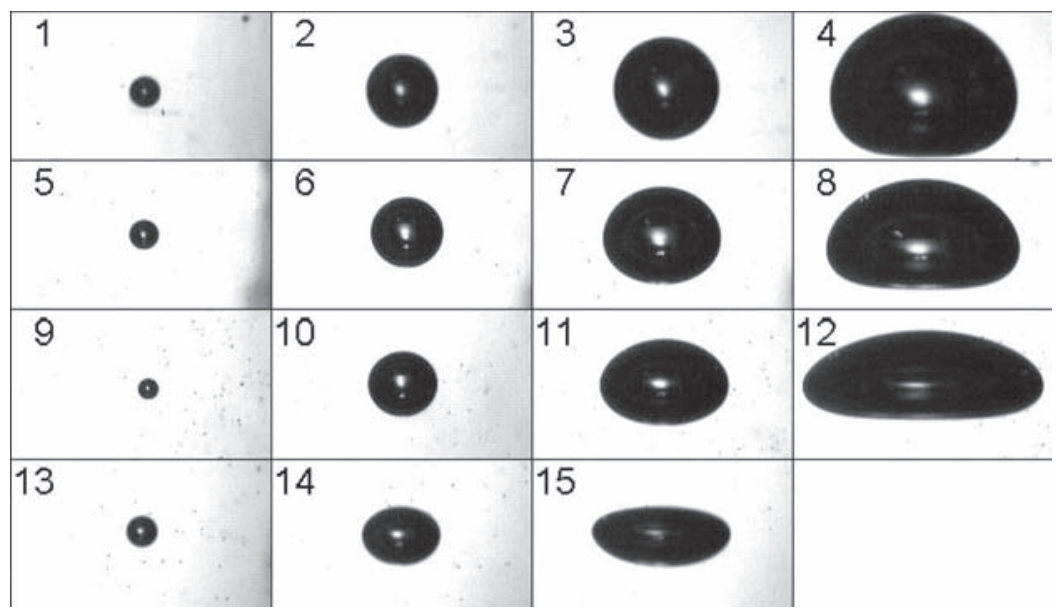

FIG. 1. The image shows the bubble shape for different sizes and fluids. The rows show experiments for the same fluid (same Morton number): first row, $M o=1.7$ (images 1-4); second row, $M o=2.9 \times 10^{-2}$ (images 5-8); third row, $M o=1.3 \times 10^{-3}$ (images 9-12); and fourth row, Mo = 4.6 $10-6$ (images 13-15). Each column shows bubbles of approximately the same size: first column, $d \approx 1.5 \mathrm{~mm}$; second column, $d \approx 3.9 \mathrm{~mm}$; third column, $d \approx 5.9 \mathrm{~mm}$; and fourth column, $d \approx 9.0 \mathrm{~mm}$. All images are shown at the same scale. The size of each frame is $\sim 14 \times 8 \mathrm{~mm}^{2}$. All liquids are water-glycerin mixtures. The properties are listed on Table I.

analysis. These measurements, via DIA, are now relatively standard for which an uncertainty of $1 \%$ is expected.

On the other hand, despite its importance in modeling, a measurement of the bubble interfacial area has not been attempted from DIA. Clearly, for large deformation, the bubbles deviate significantly from a spheroidal shape; hence, in this investigation, we measure the bubble surface area and report its variation for a wide range of parameters.

The surface area, $S$, is calculated using Pappus first theorem. ${ }^{15}$ A similar procedure, but considering the second theorem, was used by Soto et al. ${ }^{14}$ to measure the volume of bubbles with highly deformed axisymmetric shapes. The first theorem of Pappus states that the surface of revolution generated by rotating a curve $C$ about an axis of rotation is equal to the product of the arc length,

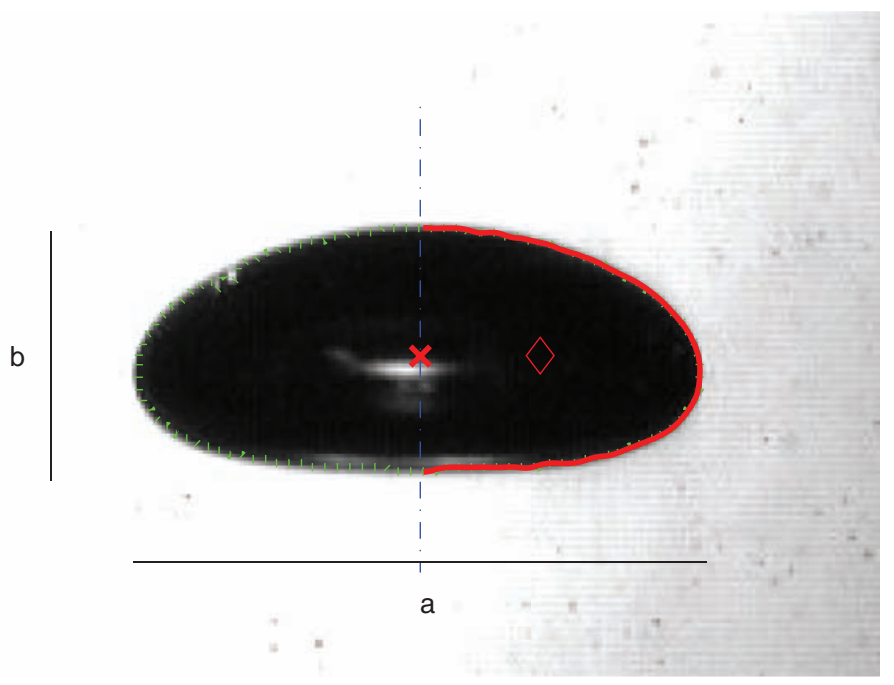

FIG. 2. Typical image of a bubble. The $\times$ shows the geometrical center of the bubble. The vertical dashed-dotted line shows the axis of symmetry. The dotted (green online) line shows the detected edge of the bubble. The continuous (red online) line shows the curve $C$ of length $s$, which is used to calculate the surface of the bubble, $S$. The $\diamond$ shows $x$ which is the centroid of $C$. For the case shown, $d=8.3 \mathrm{~mm}$ for $M o=1.3 \times 10^{-3}$. 
$s$, and the distance traveled by its geometric centroid, $x: S=2 \pi s x$. It is relatively easy to determine $C, s$, and $x$ from DIA. We used a standard edge detection algorithm to find the edge of the bubble image; from the geometric center of the bubble, the position of the vertical axis is determined. The edge is cut into two parts (left and right). From either part, the curve $C$ is obtained. Since the edge detection algorithm finds the "inner" bubble boundary (based on the maximum value of pixel intensity gradient), the position and length of $C$ have to be corrected. This is done by radially growing the curve by one pixel. We verified the accuracy of this correction by measuring the surface area of a reference sphere of known diameter. Once $C$ is known, $x$ and $s$ are easily calculated. Figure 2 shows these quantities for a typical image. This procedure was applied to all of our experiments to determine $S$ and $f_{\alpha}$. We also obtained measurements for a few data points from Huang, ${ }^{16}$ Sunol and Gonzalez-Cinca, ${ }^{17}$ and Zenit and Magnaudet. ${ }^{5}$ Since the measurement of the surface area is more sensitive to pixel-resolution error, the uncertainty of the measurement increases to $\sim 6 \%$.

\section{B. Parameter range}

The model proposed in this paper is based on the new experiments performed for this study and on many experimental measurements reported in the literature using different liquids. The corresponding range of parameters covered here is reported in the phase diagram $(M o$, Eo $)$ shown in Fig. 3. The Morton number is varied from $O\left(10^{-11}\right)$ to $O(1)$ and the Eotvos number ranges from $O\left(10^{-2}\right)$ to $O\left(10^{2}\right)$. For those ranges, the Weber number and Reynolds number are in the ranges $O\left(10^{-4}\right)$ up to $O(10)$ and $O\left(10^{-2}\right)$ to $O\left(10^{3}\right)$, respectively.

Following Rastello et al., ${ }^{18}$ we found that all data can be correlated considering $R e, W e$, and Mo. Figure 4 shows all the experimental data shown in Fig. 3 written in terms of these three dimensionless numbers. The data are closely fit to the expression ${ }^{18}$

$$
R e=2.05 \mathrm{We}^{2 / 3} \mathrm{Mo}^{-1 / 5} .
$$

Clearly, a very good correlation is found for all data. This relation is useful. Below, we propose a relation for the bubble deformation for which only the We and Mo numbers need to be known.

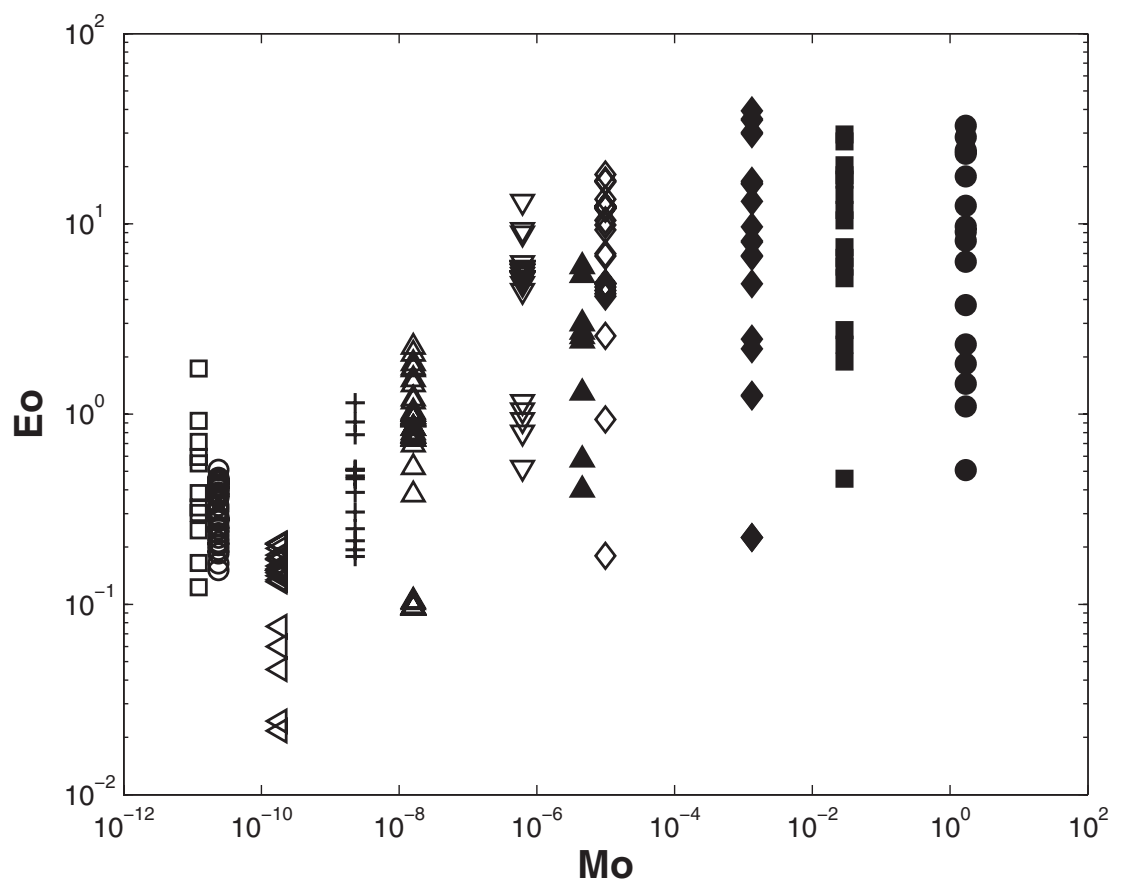

FIG. 3. Phase diagram $(M o, E o)$. Experiments from the literature considered for this study: $\circ$, ultra purified water $\left(19.6^{\circ}\right){ }^{20}$ $\square$, water $\left(\sim 28^{\circ}\right) ;{ }^{19}+$, ethanol; ${ }^{17} \triangleleft, \triangle, \nabla, \diamond$, silicon oil ${ }^{5}$ and $\boldsymbol{\Delta}, \diamond, \mathbf{\square}, \bullet$, this study. 


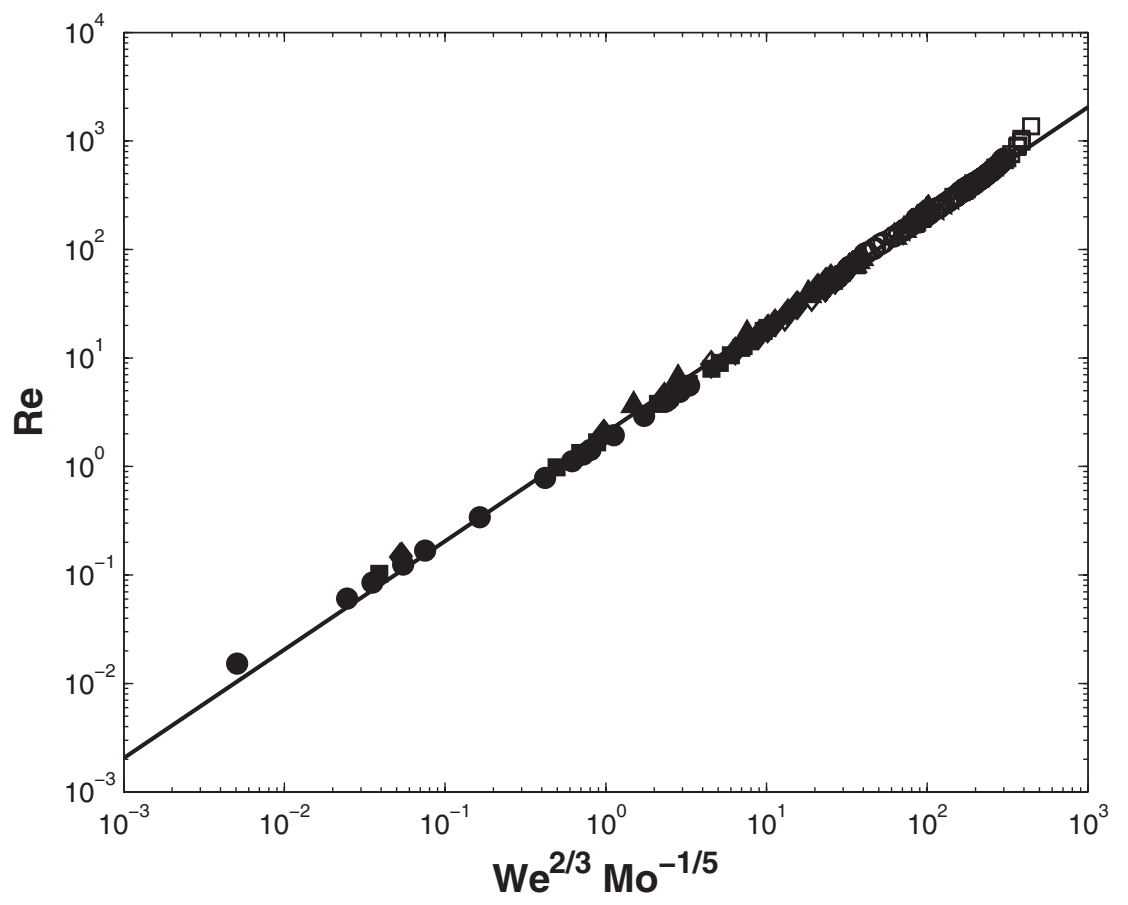

FIG. 4. Correlation between $R e, W e$, and $M o$ for all the experiments considered in this study. All symbols are the same as in Fig. 3. The solid line shows the correlation in Eq. (1).

The Morton number is defined in terms of the gravitational acceleration $g$, which limits its use to bubbles ascending as a result of buoyancy. Although, in principle, the gravitational acceleration could be replaced by the relevant acceleration in another context (either reduced or enhanced gravity), expression (1) permits us to reformulate our model in terms of We and Re only.

\section{AIR BUBBLE IN WATER}

A large number of experimental investigations of the motion of air bubbles in water have been conducted. ${ }^{1}$ In Aybers and Tapucu, ${ }^{19}$ experimental measurements for the deformation of air bubbles in water are reported for a large range of bubble diameters $(d \in[0.83 ; 7.11] \mathrm{mm})$. For this diameter range the observed shapes were spherical $(d \leq 0.83 \mathrm{~mm})$, ellipsoid without $(0.83 \leq d \leq 2.0)$ and with $(2.0 \leq d \leq 4.2)$ surface oscillation and distorted shape leading to spherical cap $(d \geq 4.2)$. In these experiments the effect of surface-active impurities was clearly demonstrated: the bubble terminal velocity was smaller than the theoretical predictions for clean bubbles.

Since water is very sensitive to impurities, Duineveld ${ }^{20}$ performed experiments in an ultra-clean environment and with high purity water for $d \in[0.66 ; 2] \mathrm{mm}$. He compared the bubble terminal velocity and deformation with the analytical solution of Moore $^{7}$ based on the potential flow solution, i.e., valid in the limit of large bubble Reynolds number. For such conditions the deformation is controlled by liquid inertia induced by the slip motion. If the distortion is small, Moore ${ }^{21}$ showed that the bubble is an oblate spheroid and that the aspect ratio is given by

$$
\chi=1+\frac{9}{64} W e .
$$

In the opposite limit of very viscous flows (i.e., high Morton numbers and low Reynolds numbers), Taylor and Acrivos ${ }^{22}$ showed that, for small inertial effects, the deformation was $\chi=1+\frac{5}{32} W e$. Note that $9 / 64 \sim 0.141$ and $5 / 32 \sim 0.156$; hence, it is difficult to experimentally observe the difference between these two behaviors since the $O(W e)$ correction is observed for $W e \leq O(1)$ corresponding to small deformation (less than $15 \%$ according to relation (2)). 
If the dynamic pressure and the surface tension pressure are balanced exactly at the stagnation points and at the intersection of the bubble surface and the horizontal plane of symmetry, the deformation can be shown to be ${ }^{21}$

$$
W e=\frac{4}{\chi^{4 / 3}} \frac{\left(\chi^{3}+\chi-2\right)}{\left(\chi^{2}-1\right)^{3}}\left[\chi^{2} \sec ^{-1} \chi-\left(\chi^{2}-1\right)^{1 / 2}\right]^{2} .
$$

Duineveld ${ }^{20}$ observed smaller deformation than that predicted by relation (3) so he proposed the following relation in order to fit his data:

$$
\chi=\sqrt{\frac{a_{1}}{W e_{0}-W e}},
$$

with $W e_{0} \simeq 4.412$ and $a_{1} \simeq-4.39$. This relation has a limited range of application since the deformation diverges as $W e \rightarrow W e_{0}$.

Loth $^{23}$ collected data from many sources in the literature to provide a new description of the aspect ratio based on the Weber and Reynolds numbers. From data for pure bubble systems over a range of Reynolds numbers from 0.2 to 5000, the author proposes the following fit:

$$
\frac{1}{\chi}=1-\left(1-E_{\min }\right) \tanh \left[c_{E} W e\right]
$$

where $E_{\min }=0.25+0.55 \exp [-0.09 R e]$ and $c_{E}=0.165+0.55 \exp [-0.3 R e]$. Note that this relation is nearly independent of the Reynolds number for $R e>20$.

The experimental results of Aybers and Tapucu ${ }^{19}$ and Duineveld ${ }^{20}$ are shown in Fig. 5(a) which also reports more recent experiments performed using high speed camera which allow a more accurate determination of the bubble shape and terminal velocity for air bubbles in tap water ${ }^{2,24}$ and ultra filtered water. ${ }^{16,25} \mathrm{We}$ also observe in this figure that the bubble deformation for all the reported experiments show the same evolution for $d \leq 1.5 \mathrm{~mm}$. For larger diameters, a different behavior is observed which could be attributed to the purity of the water. Figure 5(b) indicates that this deviation corresponds to the maximum of the terminal velocity. For $d \geq 1.5 \mathrm{~mm}$ the terminal velocity for all the experiments reported reveals the same evolution predicted by the Moore ${ }^{21}$ solution. The main reason is the decrease of bubble terminal velocity, which has been shown to be the result of interface contamination. The liquid inertia involved in bubble deformation is thus decreased and bubbles are less deformed.
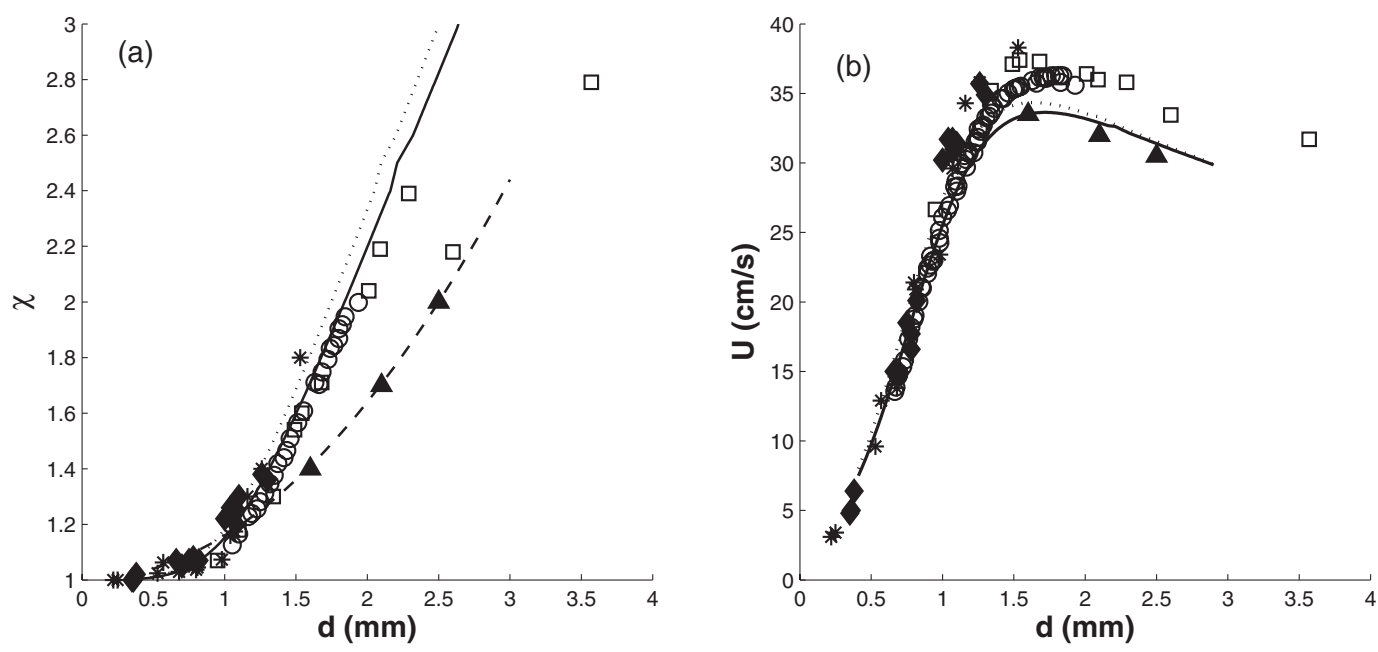

FIG. 5. Air water system. (a) Bubble aspect ratio $\chi$ and (b) terminal bubble velocity $U$ as a function of bubble equivalent diameter $d$, respectively. $\boldsymbol{\Lambda}$, tap water, ${ }^{24} \diamond$, ultra filtered water; ${ }^{25} \star$ ultra filtered water, ${ }^{29} \circ$, ultra purified water; ${ }^{20}$ and $\square$, filtered water. ${ }^{19}$ The dashed line $(---)$ shows the fit $\chi=1+0.16 d^{2}$. The solid and dotted lines show the predictions of the model proposed by Moore, ${ }^{21}$ considering physical properties at $20{ }^{\circ} \mathrm{C}$ and $25^{\circ} \mathrm{C}$, respectively. 

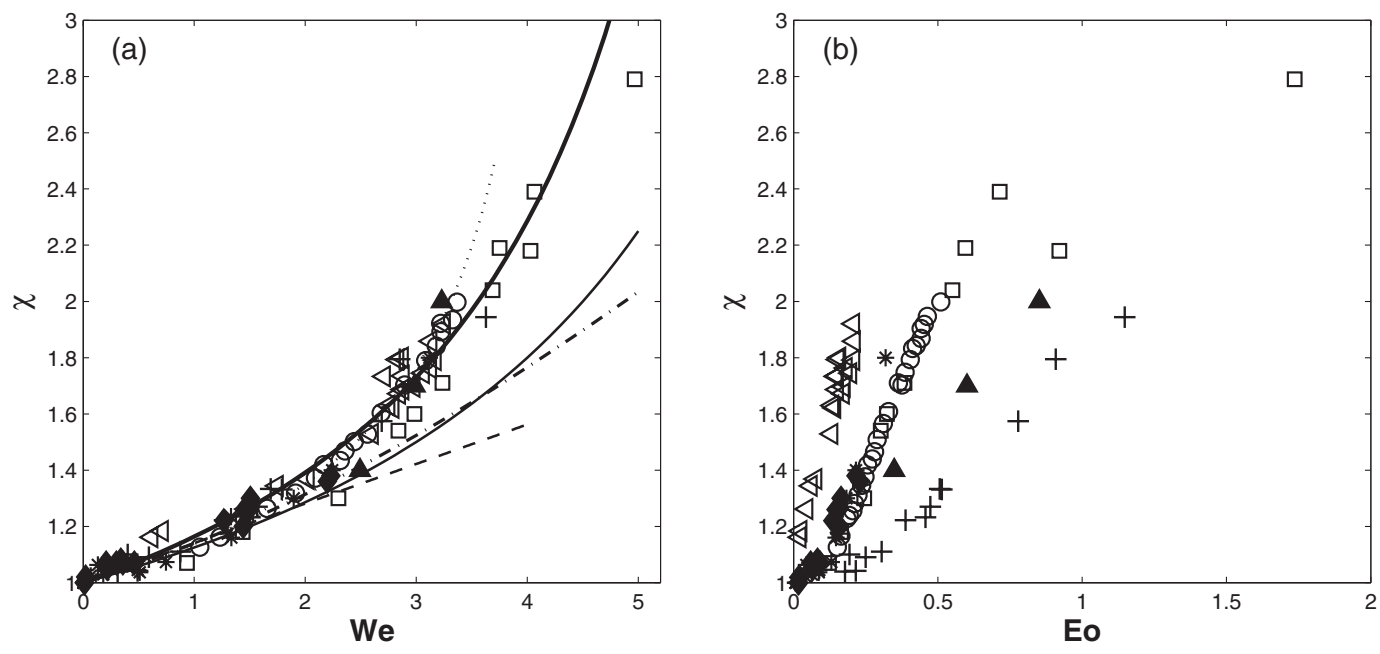

FIG. 6. Water and other low viscosity liquids. Bubble aspect ratio $\chi$ as a function of (a) Weber number $W e$ and (b) Eotvos number, Eo. $\boldsymbol{\Delta}$, tap water; ${ }^{24} \diamond$, ultra clean water, ${ }^{25} \star$, ultra filtered water; ${ }^{29} \circ$, ultra purified water; ${ }^{20} \square$, filtered water; ${ }^{19}$ ,$+\left(M o=2.3 \times 10^{-9}\right)$ ethanol, ${ }^{17}$ and $\triangleleft,\left(M o=1.8 \times 10^{-10}\right)$ silicon oil. ${ }^{5}$ The thick continuous $(-)$ and dashed $(---)$ lines show the predictions from expressions (2) and (6), respectively. The dotted (...), dashed-dotted (.,- ), and thin continuous lines show the correlations proposed by Duineveld, ${ }^{20}$ Loth, ${ }^{23}$ and Raymond and Rosant,${ }^{26}$ respectively.

Figures 6(a) and 6(b) show the deformation as function the Weber and Eotvos numbers, respectively. It is clear from these plots that the relevant dimensionless number to be used to describe the deformation is the Weber number. Indeed, when deformation is reported versus the Weber number all the data collapse on the same curve (Fig. 6(a)); on the other hand, considering the Eotvos number the data are greatly scattered.

It is also very interesting to note that (i) clean and contaminated bubbles also follow the same evolution and (ii) the experiments of Aybers and Tapucu ${ }^{19}$ corresponding to various bubble shapes including spherical cap also follow the same trend. The deformation evolution also appears not to be significantly affected by the nature of the trajectory. Ellingsen and Risso ${ }^{2}$ and Riboux et al. ${ }^{24}$ have shown that the velocity remains aligned with the small axis when the bubble experiences a helical trajectory.

As shown in Fig. 6, the following simple relation closely describes the bubble deformation for water for $\chi$ up to 3 :

$$
\chi=\frac{1}{1-\frac{9}{64} W e} .
$$

In the limit of small deformation (i.e., small $W e$ ) this relation is in accordance with expression (2), which is valid up to $W e \approx 2$. Relation (5) is also shown in the figure, for the limit of large Reynolds numbers. This relation closely predicts the deformation up to $\chi \approx 2.5$. Raymond and Rosant ${ }^{26}$ proposed a relation, which is very similar to Eq. (6) but based on larger Morton number experiments. The agreement with this expression is also good for small deformation. However, for both cases, for $W e>2$, the predicted deformation is below the experimental data shown in Fig. 6. The source of this difference with the deformation measured in water systems is discussed in Sec. V considering larger Morton number.

\section{EFFECT OF MORTON NUMBER}

Let us now consider the bubble deformation for more viscous fluids in order to cover a wider range of Morton numbers (see Fig. 3). We first observed that the evolution of the deformation with the Weber number was not affected significantly by the change in Morton number for $M o \leq 2 \times 10^{-9}$. Indeed, as shown in Fig. 6(a), the evolution of the aspect ratio for the silicon oil 


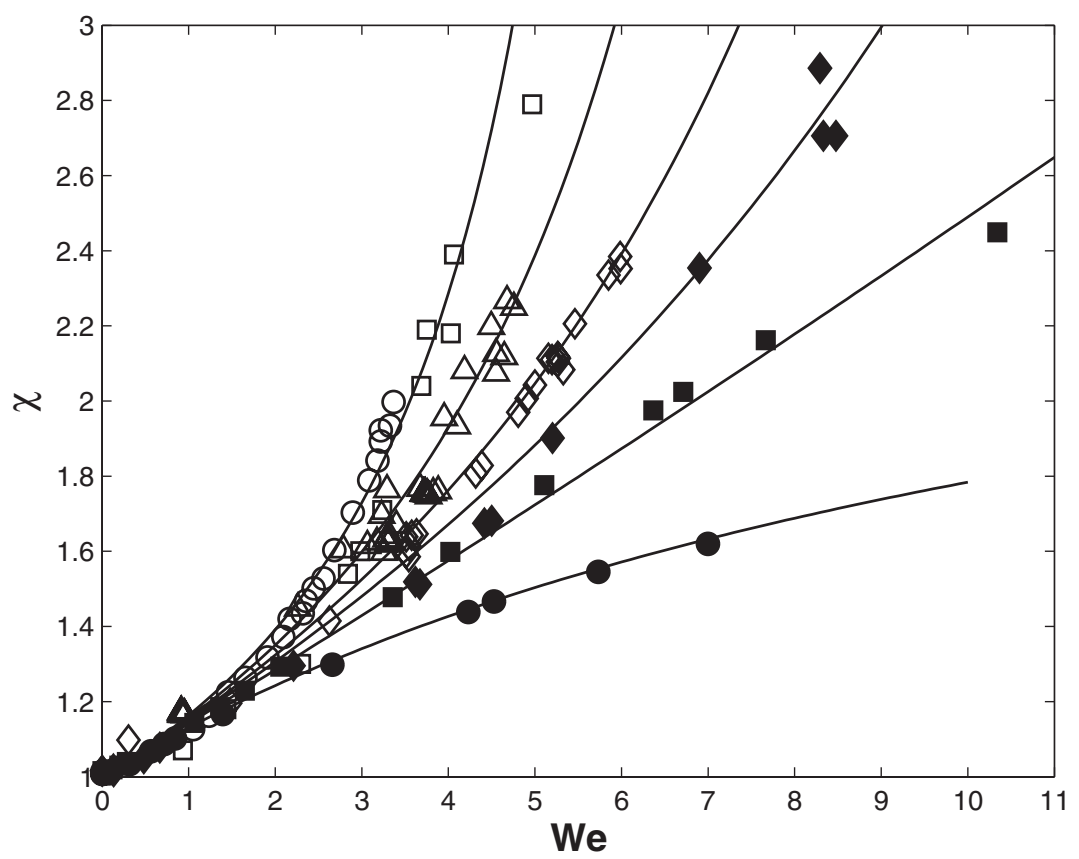

FIG. 7. Effect of Weber and Morton numbers on bubble deformation. o, Ultra purified water; ${ }^{20} \square$, filtered water; ${ }^{19}$ $\diamond\left(M o=9.9 \times 10^{-6}\right)$ and $\Delta\left(M o=1.6 \times 10^{-8}\right)$ silicon oils, ${ }^{5}$ and $\diamond\left(M o=1.3 \times 10^{-3}\right), \mathbf{\square}\left(M o=2.9 \times 10^{-2}\right)$, - $(M o=1.7)$, water-glycerin mixtures (this study). The solid lines show the prediction of Eq. (7), considering Eq. (8), for the corresponding Morton numbers of each experiment.

$\left(M o=1.8 \times 10^{-10}\right)$ and for ethanol $\left(M o=2.3 \times 10^{-9}\right)$ are very close to that observed for water. The evolution as a function of the Eotvos number, shown in Fig. 6(b), confirms that the correct dimensionless number for these liquids is the Weber number.

Figure 7 shows the effect of larger Morton numbers on the deformation. For comparison, the results of Aybers and Tapucu ${ }^{19}$ and Duineveld ${ }^{20}$ (which correspond to an air-water system) are used to show the water-like evolution observed up to Morton number Mo $\approx 2 \times 10^{-9}$. The bubble deformation obtained in the present study for a water-glycerol mixture $(M o=1.68$, $\left.M o=2.9 \times 10^{-2}, M o=1.3 \times 10^{-3}\right)$ and by Zenit and Magnaudet ${ }^{5}$ for two silicon oils $(M o=1.6$ $\times 10^{-8}$ and $\left.M o=9.9 \times 10^{-6}\right)$ are also shown in Fig. 7. The deformation is observed to decrease monotonically with the Morton number.

A detailed inspection of the data, by considering the evolution of $1-1 / \chi$, reveals that the following simple expression closely predicts the bubble aspect ratio for all the fluids considered here:

$$
\chi=\frac{1}{1-\frac{9}{64} W e(1+K(M o) W e)^{-1}},
$$

where $K(\mathrm{Mo})$ is a function of the Morton number only. In order to recover relation (6), valid for water and other low viscosity liquids, which is consistent with the analytical solution of Moore ${ }^{21}$ for small deformation, the condition $\lim _{M o \rightarrow 0} K(M o)=0$ must be satisfied. Our data suggest that $K(M o)$ evolves approximately as $M o^{1 / 10}$. This dependence can be closely fitted by the following simple expression:

$$
K(M o)=0.2 M o^{1 / 10}
$$

The prediction of expression (7), considering also Eq. (8), is compared with the experimental measurements in Fig. 7. An excellent agreement is observed. Additionally, we compared our results with the recent experimental measurements of Rastello et al. ${ }^{18}$ leading also to a very close agreement 
(not shown in Fig. 7 for clarity). We also observed a very good agreement with the predictions of Loth's correlation $^{23}$ for larger Morton number than those reported in Fig. 6. For Mo $>10^{-3}$ we found some differences with the data reported by Raymond and Rosant. ${ }^{26}$ Both our experiments and model, result in smaller bubble deformation for a given value of $W e$, particularly for $W e>2.5$. It is not easy to assess what is the cause of these discrepancies. In general, we think that differences between data sets may arise from the deformation measurement. The validity of our results rests in the good agreement with the results reported by Loth $^{23}$ and Rastello et al. ${ }^{18}$ for such Mo values.

In this paper, we show two regimes for the bubble deformation. For Morton number smaller than $10^{-9}$ we observe a water-like behavior nearly independent on the Morton number and in good agreement with recent different sources ${ }^{5,17,20,24}$ as well as with classic data. ${ }^{19,20}$ In most cases, the degree of cleanliness of the bubble surface is not controlled but all these experiments show deformation in agreement with the model proposed in this study whatever the surface contamination level. When the Morton number increases, we observe less deformation than the water-like behavior as clearly shown in Fig. 7 in agreement with the recent experiments of Rastello et al. ${ }^{18}$ as well as with the empirical model of Loth. ${ }^{23}$ As discussed above, neither surface contamination nor bubble trajectory is expected to produce less deformation. Therefore, other physical mechanisms must be analyzed to further understand this behavior. It can be argued that the viscous boundary layer developing at the bubble surface can be responsible for the reduced deformation for a given Weber number when increasing the Morton number. As shown by Kang and Leal, ${ }^{27}$ the bubble Reynolds number has a significant effect on the pressure distribution at a spherical bubble surface. Same effect is, of course, expected for deformed bubble, most likely resulting in less deformation for a given Weber number. We plan to further pursue this argument via numerical simulations in a future study.

\section{BUBBLE SURFACE AREA}

A direct application of the results presented in this paper is their use to describe the interfacial area $\alpha_{I}$. This quantity is often used in the Eulerian two-fluid models to account for the interfacial momentum, energy and mass exchange between the phases. In mono-dispersed bubbly flows, the interfacial area is a function of the gas void fraction $\alpha_{G}$, the volume $V$ and the surface $S$ of the bubbles:

$$
\alpha_{I}=\alpha_{G} \frac{S}{V}
$$

As shown above, the bubble shape and, therefore, the interfacial area change significantly with both the Morton number and the Weber number. This parameter can vary significantly, even within the same flow, in a complex system. To our knowledge, experimental measurements of this parameter have not been reported to date; only a few numerical studies exists ${ }^{26,28}$ but their results have not been verified experimentally.

For a spherical bubble, $\alpha_{I}=6 \alpha_{G} / d_{e q}$. When the bubble deforms, according to Figueroa and Legendre, ${ }^{13}$ we can express the interfacial area as

$$
\alpha_{I}=\alpha_{G} \frac{6}{d} f_{\alpha}(\chi)
$$

where $f_{\alpha}$ is a measure of the relative increase of the interfacial area with the deformation.

For a spheroidal bubble of aspect ratio $\chi$, an expression for $f_{\alpha}$ can be readily obtained: ${ }^{21}$

$$
f_{\alpha}(\chi)=\left[\frac{\chi^{2 / 3}}{2}+\frac{1}{4 \chi^{1 / 3} \sqrt{\chi^{2}-1}} \operatorname{Ln}\left(\frac{\chi+\sqrt{\chi^{2}-1}}{\chi-\sqrt{\chi^{2}-1}}\right)\right] .
$$

From digital image analysis, described in Sec. III, a measurement of the surface area, $S$, of a given bubble can be obtained. Despite its importance, surprisingly, a direct measurement of the surface area of bubbles has not been reported to date. We can directly calculate $f_{\alpha}$ for a wide range of deformations.

Figure 8 shows the measured value of $f_{\alpha}$ for all the experiments conducted in this study. For the largest deformation considered here $(\chi \approx 3)$ the increase of the interfacial area is significant since it 


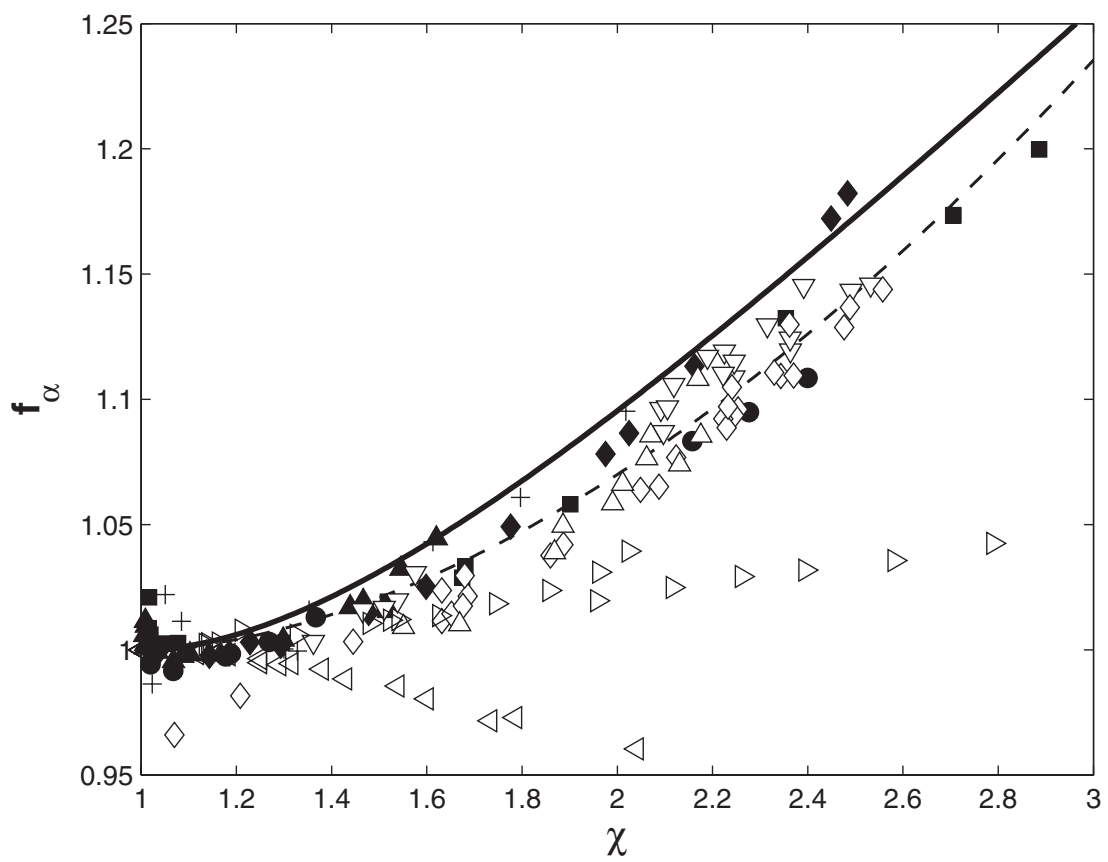

FIG. 8. Normalized bubble surface, $f_{\alpha}$, as a function of bubble aspect ratio. Measurements for: $\Delta\left(M o=1.6 \times 10^{-6}\right)$, $\checkmark\left(M o=1.32 \times 10^{-3}\right), \boldsymbol{\square}(M o=0.029), \bullet(M o=1.68)$, water-glycerin mixtures (this study). For comparison some other data are shown: Huang et al. ${ }^{29}\left(\star\right.$, Mo $\left.=2.6 \times 10^{-10}\right)$; Sunol and Gonzalez-Cinca ${ }^{17}\left(+\right.$, Mo $\left.=2.3 \times 10^{-9}\right)$; Lai et al. $^{28}$ $\left(\triangleright, 2.0<M o<7.7 \times 10^{-7}\right)$; and Raymond and $\operatorname{Rosant}^{26}\left(\triangleleft, 2.0<M o<7.7 \times 10^{-7}\right)$. Shown also are the data of Zenit and Magnaudet ${ }^{5}$ for silicon oils: $\diamond\left(M o=9.9 \times 10^{-6}\right), \nabla\left(M o=6.2 \times 10^{-7}\right)$, and $\triangle\left(M o=1.6 \times 10^{-8}\right)$. The solid and dashed lines show the predictions of Eqs. (10) and (11), respectively.

can reach $20 \%$ for $\chi=3$. The measurements are contrasted with the prediction from expression (10). Clearly, the agreement is good, for the whole range of Morton numbers and deformations but the expression overestimates the increase of the surface area for most values of $\chi$. The following fit,

$$
f_{\alpha}=1+0.07(\chi-1)^{7 / 4}
$$

is shown in the figure. It is simple and closely fits the evolution of the surface increase for the range of Morton number considered in this study. Nevertheless, despite the wide range of deformations, the interfacial area can be approximated by assuming an ellipsoidal shape. Finally, using relations (9) and (10) or (11) coupled with (7), it is possible to have a good description of the interfacial area for a wide range of fluid properties and deformation. An explicit dependence with $W e$ and Re can also be obtained considering Eq. (1).

In the figure, the simulation results from Raymond and Rosant ${ }^{26}$ and Lai et al. ${ }^{28}$ are also shown. The results of Lai et al. ${ }^{28}$ show an increasing value of the normalized bubble surface as the aspect ratio increases, in accordance with the experimental measurements; however, their computed results are below the rest of the data. Such underestimation of the bubble surface may result from the particular simulation technique used in their study. The results of Raymond and Rosant ${ }^{26}$ show a very different trend: they report that the bubble surface decreases with aspect ratio. These results are, most likely, flawed. For a spherical bubble $(\chi=1)$ the surface is minimized; hence, as deformation increases, the surface of the bubble should increase.

\section{CONCLUDING REMARKS}

The bubble deformation has been analyzed for different gas-liquid systems. For the air-water case, the relevant parameter to describe the deformation is the Weber number. By considering only this number, the bubble deformation can be described universally for all bubble shapes, contamination 
levels and path trajectories. A water-like behavior is observed for low viscosity fluids for Morton numbers of up to $M o$ of $O\left(10^{-9}\right)$. For more viscous liquids the deformation for a given Weber number decreases with the Morton number. The relation obtained for small Morton numbers was extended for more viscous liquids, considering a simple correction to account for variations on both Weber and Morton numbers. This study indicates that the bubble deformation can be fully described by only knowing $W e$ and Mo. This result is, in fact, consistent with what is expected from a simple dimensional analysis of the system.

The evolution of the bubble surface area has also been briefly discussed. A simple model has been proposed to describe the evolution of the interfacial area for moderately deformed bubbles. With such a model it is possible to have a dynamic representation of the bubble deformation. Thus in regions where the Weber number is reduced because of a smaller slip velocity (for example in recirculation zone in a downcomer), the interfacial area should decrease. The simple relation proposed here is of interest for practical applications and for CFD tools where bubble deformation has to be characterized since it directly affects the surface of exchange in many flow configurations.

${ }^{1}$ R. Clift, J. R. Grace, and M. E. Weber, Bubbles, Drops and Particles (Academic, New York, 1978).

${ }^{2} \mathrm{~K}$. Ellingsen and F. Risso, "On the rise of an ellipsoidal bubble in water: oscillatory paths and liquid-induced velocity," J. Fluid Mech. 440, 235 (2001).

${ }^{3}$ T. Sanada, M. Shirota, and M. Watanabe, "Bubble wake visualization by using photochromic dye," Chem. Eng. Sci. 62, 7264 (2007).

${ }^{4}$ G. Mougin and J. Magnaudet, "Wake instability of a fixed spheroidal bubble," J. Fluid Mech. 572, 311 (2007).

${ }^{5} \mathrm{R}$. Zenit and J. Magnaudet, "Path instability of rising spheroidal air bubbles: A shape controlled process," Phys. Fluids 20, 061702 (2008).

${ }^{6}$ B. Yang and A. Prosperetti, "Linear stability of the flow past a spheroidal bubble," J. Fluid Mech. 582, 53 (2007).

${ }^{7}$ D. W. Moore, "The rise of a gas bubble in a viscous liquid," J. Fluid Mech. 6, 113 (1959).

${ }^{8}$ T. Maxworthy, C. Gnann, M. Kurten, and F. Durst, "Experiments on the rise of air bubbles in clean viscous liquid," J. Fluid Mech. 321, 421 (1996).

${ }^{9}$ A. Tomiyama, I. Kataoka, I. Zun, and T. Sakaguchi, "Drag coefficients of single air bubbles under normal and micro gravity conditions," JSME Int. J., Ser. B 41, 472 (1998).

${ }^{10}$ D. Legendre, "On the relation between the drag and the vorticity produced on a clean bubble," Phys. Fluids 19, 018102 (2007).

${ }^{11}$ A. C. Lochiel and P. H. Calderbank, "Mass transfer in the continuous phase around axisymmetric bodies of revolution," Chem. Eng. Sci. 19, 471 (1964).

${ }^{12}$ M. Favelukis and C. H. Ly, "Unsteady mass transfer around spheroidal drops in potential flow," Chem. Eng. Sci. 60, 7011 (2005).

${ }^{13}$ B. Figueroa and D. Legendre, "Mass or heat transfer from spheroidal gas bubbles rising through a stationary liquid," Chem. Eng. Sci. 65, 6296 (2010).

${ }^{14}$ E. Soto, C. Goujon, R. Zenit, and O. Manero, "A study of velocity discontinuity for single air bubbles rising in an associative polymer," Phys. Fluids 18, 121510 (2006).

${ }^{15}$ E. W. Weisstein, CRC Concise Encyclopedia of Mathematics (CRC, Boca Raton, FL, 1998).

${ }^{16} \mathrm{Z}$. Huang, "Efficacité de capture dans les procédés de flottation," Ph.D. dissertation (Institut National des Sciences Appliquées de Toulouse, Toulouse, France, 2009).

${ }^{17}$ F. Sunol and R. Gonzalez-Cinca, "Rise, bouncing and coalescence of bubbles impacting at a free surface," Colloids Surf., A $\mathbf{3 6 5}, 36$ (2010).

${ }^{18}$ M. Rastello, J.-L. Marié, and M. Lance, "Drag and lift forces on clean spherical and ellipsoidal bubbles in a solid body rotating flow," J. Fluid Mech. 682, 434 (2011).

${ }^{19}$ N. M. Aybers and A. Tapucu, "Studies on the drag and shape of gas bubbles rising through a stagnant liquid," Wärme-Stoffübertrag. 2, 171 (1969).

${ }^{20}$ P. C. Duineveld, "The rise velocity and shape of bubbles in pure water at high Reynolds number," J. Fluid Mech. 292, 325 (1995).

${ }^{21}$ D. W. Moore, "The velocity of rise of distorted gas bubbles in a liquid of small viscosity," J. Fluid Mech. 23, 749 (1965).

${ }^{22}$ T. D. Taylor and A. Acrivos, "On the deformation and drag of a falling viscous drop at low Reynolds number," J. Fluid Mech. 18, 466 (1964).

${ }^{23}$ E. Loth, "Quasi-steady shape and drag of deformable bubbles and drops," Int. J. Multiphase Flow 34, 523 (2008).

${ }^{24}$ G. Riboux, F. Risso, and D. Legendre, "Experimental characterization of the agitation generated by bubbles rising at high Reynolds number," J. Fluid Mech. 643, 509 (2010).

${ }^{25}$ V. Sarrot, Z. Huang, D. Legendre, and P. Guiraud, "Experimental determination of particles capture efficiency in flotation," Chem. Eng. Sci. 62, 7359 (2007).

${ }^{26} \mathrm{~F}$. Raymond and J.-M. Rosant, "A numerical and experimental study of the terminal velocity and shape of bubbles in viscous liquid,” Chem. Eng. Sci. 55, 943 (2000). 
${ }^{27}$ I. S. Kang and L. G. Leal, "The drag coefficient for a spherical bubble in a uniform streaming flow," Phys. Fluids 31, 233 (1988).

${ }^{28}$ H. Lai, Y. Y. Yan, and C. R. Gentle, "Calculation procedure for conjugate viscous flows about and inside single bubbles," Numer. Heat Transfer, Part B 43, 241 (2003).

${ }^{29}$ Z. Huang, D. Legendre, and P. Guiraud, "A new experimental method for determining particle capture efficiency in flotation,” Chem. Eng. Sci. 66, 982 (2011). 